\title{
Alcoholic Hepatitis with and without Alcoholic Hyaline
}

\author{
Shozo Nakamura, Yoshiaki Takezawa,* Takashi Sato,* \\ Tatsuya Aikawa $\dagger$ and Kryoshi Kera $\dagger$ \\ Tohoku University Health Center, Sendai 980, *The First \\ Department of Internal Medicine, Tohoku University School of \\ Medicine, Sendai 980, and †Section of Gastroenterology, Jusendo \\ General Hospital, Koriyama 963
}

\begin{abstract}
Nakamura, S., Takezawa, Y., Sato, T., Alkawa, T. and Krea, K. Alcoholic Hepatitis with and without Alcoholic Hyaline. Tohoku J. exp. Med., 1977,123 (4), 351-358 - Sixteen cases of alcoholic hepatitis with alcoholic hyaline (Group I) and 13 cases without alcoholic hyaline (Group II) have been collected since 1970. These were most frequently found in the fifth decade in both groups. One female was found in Group 1. Cases of about two-thirds of both groups were consumers of $110 \mathrm{~g}$ or more of alcohol per day. No significant differences except fever and erythrocyte sedimentation rate were found in clinical and laboratory changes between both groups. Fatty change in liver biopsy specimens were more frequently seen in Group I than in Group II. The wedged hepatic venous pressure was markedly elevated in alcoholic hepatitis with cirrhosis and moderately elevated in alcoholic hepatitis without cirrhosis. Wedged hepatic venography showed the main portal trunk and extrahepatic collaterals, namely, reversal of the portal flow or such tendency in 2 out of 5 patients of Group $I$. alcoholic hepatitis; alcoholic hyaline; wedged hepatic venous pressure; reverse portal blood flow
\end{abstract}

Recently consumption of alcohol (ethanol) has increased in most countries in the world. In Japan, average consumption of alcohol, which was 2.6 liters per capita per year in 1960, increased to 5.0 liters per capita in 1974. This is the largest in Japanese history. Though it is not so large as compared with the United States or many European countries, it is large enough to cause alcoholic liver diseases. In proportion to the increase in consumption of alcohol in Japanese, patients with alcoholic liver diseases have increased, too. Patients with alcoholic liver cirrhosis who were admitted to the First Department of Internal Medicine, Tohoku University Hospital, amounted to $21 \%$ of the total cirrhotic patients in $1951-60,25 \%$ in $1961-65,42 \%$ in $1966-70$, and $47 \%$ in $1971-75$.

Alcoholic hepatitis with alcoholic hyaline, which had been thought to be very rare in Japan (Nakamura et al. 1967), was seen by us not rarely since 1970. Cases of alcoholic hepatitis with alcoholic hyaline hitherto seen by us amounted to 16. This figure is not so large as in the United States or European countries, but one of the largest in Japan (Takada 1976).

Received for publication, June 6, 1977. 
In the present paper, cases of alcoholic hepatitis with and without alcoholic hyaline were clinically and morphologically studied.

\section{Materials and Methods}

The material consists of $\mathbf{1 6}$ cases of alcoholic hepatitis with alcoholic hyaline and 13 cases of alcoholic hepatitis without alcoholic hyaline. They were admitted either to the First Department of Internal Medicine, Tohoku University Hospital, or to the Section of Gastroenterology, Jusendo General Hospital in the period from 1970 to 1975.

Alcoholic hepatitis was diagnosed when the patient had history of excessive intake of $66 \mathrm{~g}$ or more of alcohol ( $3 \mathrm{go}$ or more of sake) per day and liver function disturbances and also it was confirmed by liver biopsy. The criteria for histological diagnosis was to show parenchymal necrosis and predominantly polymorphonuclear leucocytic inflammation. It did not necessarily include steatosis, fibrosis, or cirrhosis.

The patients were classified into 2 groups on the basis of the presence or absence of alcholic hyaline in their biopsy specimens: 16 patients with alcoholic hyaline (Group I) and 13 patients without alcoholic hyaline (Group II). In these two groups the clinical, laboratory and histological features were compared. Hepatic circulatory changes were studied in some of the patients. The wedged hepatic venous pressure was measured in 8 patients of Group I and 2 patients of Group II. Wedged hepatic venography was carried out in 5 patients of Group I. It was performed by injecting rapidly by hand $20 \mathrm{ml}$ of a contrast medium into the wedged hepatic venous catheter. A film was taken near the end of the injection, or serial films were exposed during and after the injection.

\section{Results}

Age and sex. Average age was 43.3 years (range: $29-59$ years) in Group I and 51.5 years (range: $24-67$ years) in Group II. It was most frequently seen in the fifth decade in both groups. There was one female, geisha, in Group I.

Alcoholic intake. Daily alcoholic intake was $118 \mathrm{~g}$ (range: 66-243 g) in Group I and $130 \mathrm{~g}$ (range: $66-220 \mathrm{~g}$ ) in Group II. The difference was not statistically significant. In both groups about two-thirds of the patients were individuals who consumed $110 \mathrm{~g}$ or more of alcohol (5 go or more of sake) daily. Sake (a Japanese rice wine containing $15-16 \%$ of alcohol) was the most popular alcoholic beverage among the patients in both groups. Only 2 patients of Group I and 1 of Group II consumed whisky, and 1 of Group I beer. None preferred shochu, a Japanese spirits. Duration of alcohol abuse averaged 18.6 years (range: 6-30 years) in Group I and 32.2years (range: 6-47 years) in Group II. The difference was statistically significant $(p<0.0005)$. Abstinence period of one month or more was observed in $85 \%$ of Group I but in none of Group II. The difference was statistically significant $(p<0.0005)$.

Clinical findings. Jaundice was observed in $67 \%$ of patients of Group I and in $54 \%$ of Group II. Hepatomegaly was found in $88 \%$ of Group I and in $77 \%$ of Group II. Splenomegaly and ascites were seen in $7 \%$ and $20 \%$ of Group I, respectively, but in none of Group II. Fever was noted in $50 \%$ of Group I, but in none of Group II. The difference of incidence of fever between both groups was statistically significant $(p<0.05)$.

Laboratory tests. Glutamic oxaloacetic transaminase (GOT) was elevated in 


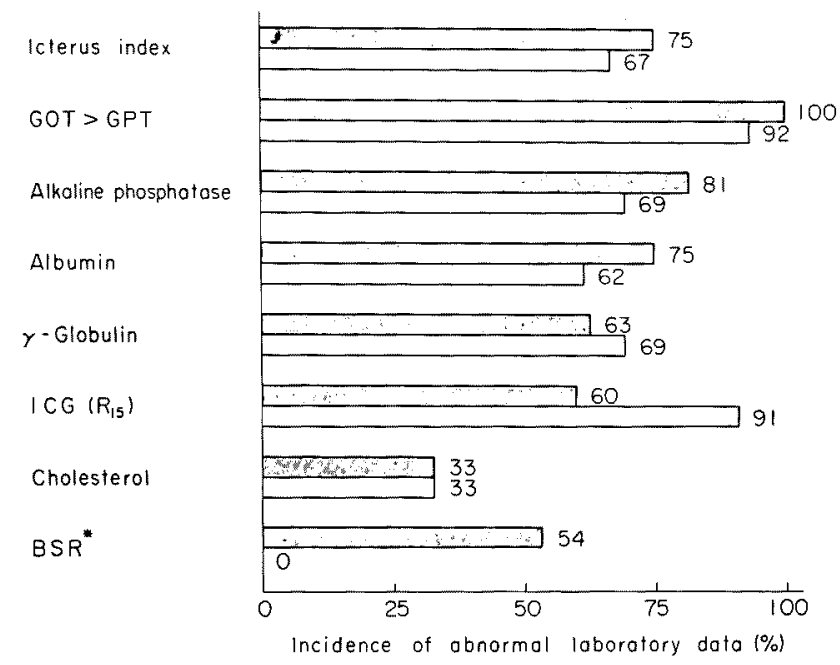

Fig. 1. Laboratory tests in alcoholic hepatitis with alcoholic hyaline (hatched column) and without alcoholic hyaline (open column). An asterisk $\left(^{*}\right)$ indicates statistically significant difference at 5 per cent level.

$94 \%$ of Group I and in all patients of Group II, but increases over 400 Karmen units were observed only in $6 \%$ of Group I and in $15 \%$ of Group II. GOT was higher than glutamic pyruvic transaminase (GPT) in all but one patient of Group I (Fig. 1). GPT was increased in $81 \%$ of Group I and in all patients of Group II. Abnormalities in icterus index or serum bilirubin, alkaline phosphatase, indocyanine green retention, serum albumin, and serum gamma-globulin were observed in about two-thirds or more of the patients in both groups (Fig. 1). Leucocytosis (leucocyte count above 10,000 per $\mu 1$ ) was found in $18 \%$ of Group I, but in none of Group II. Erythrocyte sedimentation rate was elevated (20 mm/hr or more) in $54 \%$ of Group I, but in none of Group II. The difference in incidence of elevated erythrocyte sedimentation rate between both groups was statistically significant $(p<0.05)$.

Liver biopsy. Alcoholic hyaline was seen in the biopsies of 16 patients (Group I) and was absent from the remaining 13 (Group II). It was shown as irregular eosinophilic coarsely granular mass in the enlarged vacuolated cytoplasm of liver cells when stained with hematoxylin and eosin (Figs. 2 and 3). With Mallory's azan stain, typical alcoholic hyaline appeared as a blue structure. Liver cells containing alcoholic hyaline were surrounded by neutrophil polymorphonuclear leucocytes, but infiltration of leucocytes around the liver cells was not marked.

Focal, zonal, or submassive necrosis of liver cells was present as shown in Fig. 4. Fatty change was significantly more frequently seen in Group I than in Group II $(p<0.05 ;$ Figs. 2 and 4$)$. Cirrhosis, pericellular fibrosis, and central vein fibrosis also seemed to be more frequent in Group I than in Group II, but the differences were not statistically significant (Fig. 4). No differences in incidence of acidophilic bodies, cholestasis, and inflammatory change around the central veins 


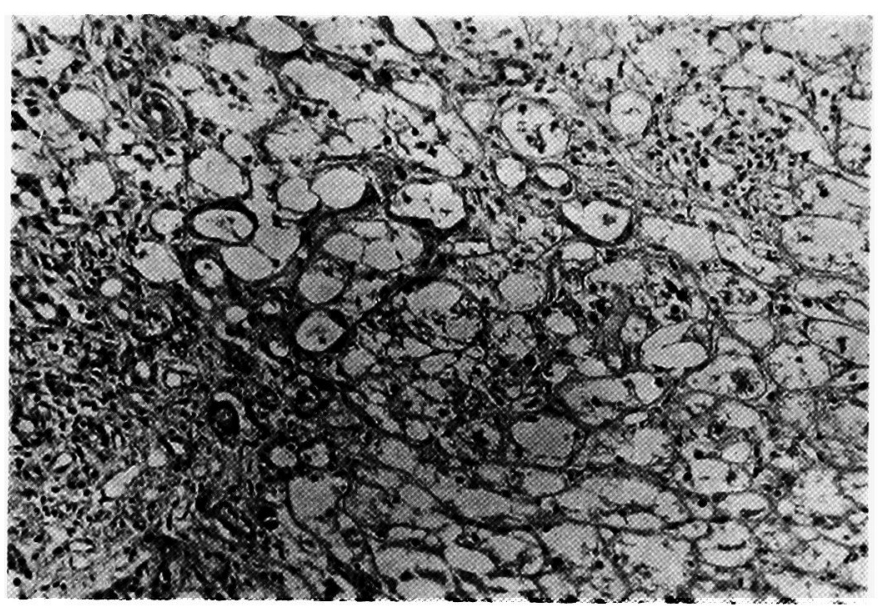

Fig. 2. Liver biopsy of a female patient with alcoholic hepatitis (Group I). Mallory's azan stain. There are alcoholic hyaline, swollen liver cells, fatty metamorphosis, hepatocellular necrosis, inflammatory infiltration, proliferation of bile ducts, fibrosis, and cholestasis.

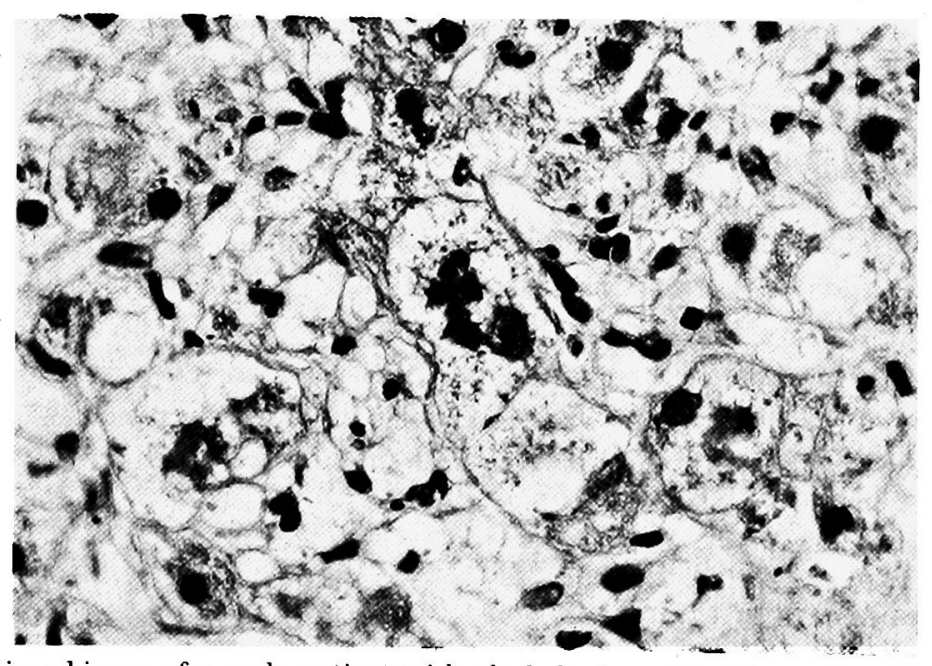

Fig. 3. Liver biopsy of a male patient with alcoholic hepatitis (Group I). Hematoxylir and eosin stain. Alcoholic hyaline is clearly seen in swollen liver cells, especially in the center. Fat vacuoles are also observed. Polymorphonuclear leucocytic infiltration is not marked.

were observed in both groups.

Wedged hepatic venous pressure. The wedged hepatic venous pressure of 8 patients of Group I averaged $261 \mathrm{~mm} \mathrm{H}_{2} \mathrm{O}$. When they were subdivided into cirrhotic and non-cirrhotic subgroups, the wedged hepatic venous pressure of 4 cirrhotic patients averaged $315 \mathrm{~mm} \mathrm{H}_{2} \mathrm{O}$ (range: $290-380 \mathrm{~mm} \mathrm{H}_{2} \mathrm{O}$ ) and that of 4 non-cirrhotic patients $207 \mathrm{~mm} \mathrm{H}_{2} \mathrm{O}$ (range: 110-340 $\mathrm{mm} \mathrm{H}_{2} \mathrm{O}$ ). In Group II, 


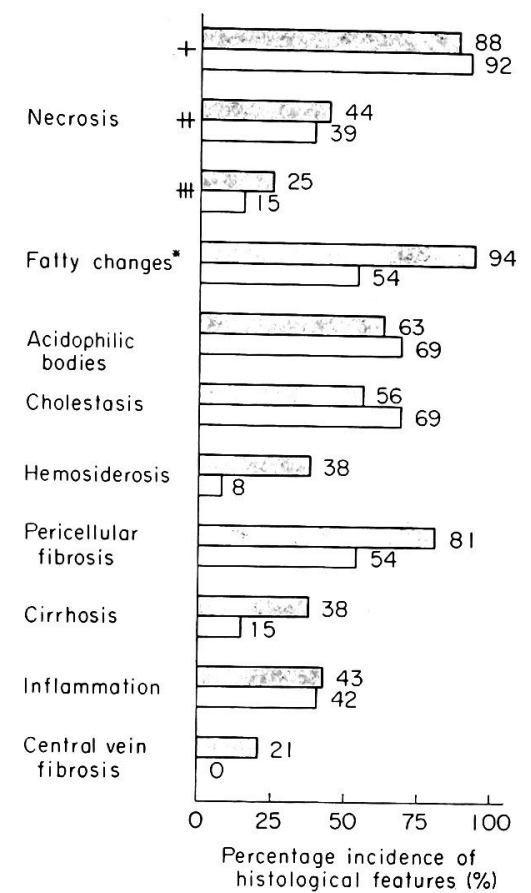

Fig. 4. Histological changes in alcoholic hepatitis with alcoholic hyaline (hatched column) and without alcoholic hyaline (open column). An asterisk (*) indicates statistically significant difference at 5 per cent level.

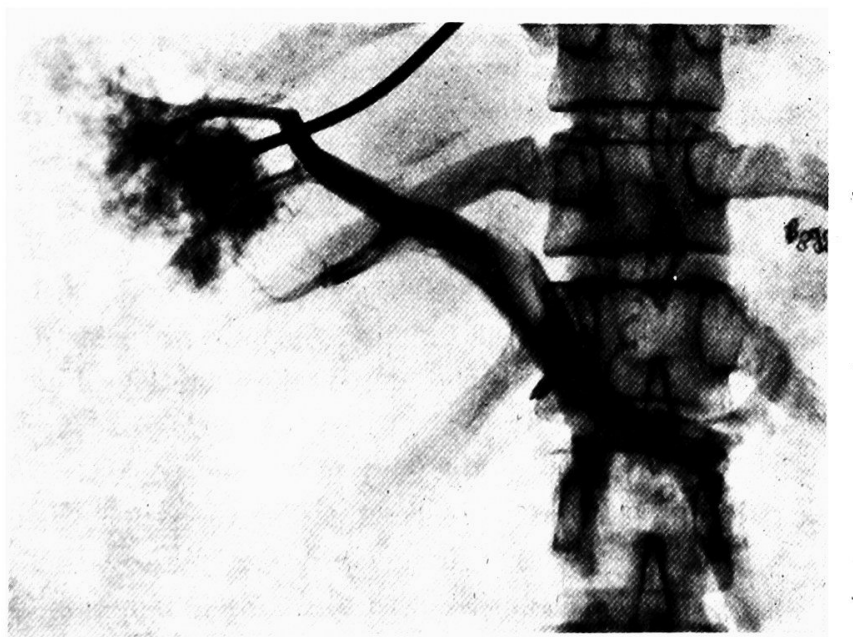

Fig. 5. Wedged hepatic venogram of a female patient with alcoholic hepatitis and cirrhosis. The main portal trunk, the inferior mesenteric vein, and the gastric coronary vein are revealed. The contrast medium in the main portal trunk is diluted with the blood flowing from the left portal vein, showing clearly the reversal of the portal blood flow. 
the wedged venous pressure in a patient with cirrhosis was $377 \mathrm{~mm} \mathrm{H} \mathrm{H}_{2} \mathrm{O}$ and that in a patient without cirrhosis $150 \mathrm{~mm} \mathrm{H}_{2} \mathrm{O}$.

Wedged hepatic venography. In 2 of the 5 patients of Group I in whom wedged hepatic venography was carried out, both the main portal trunk and extrahepatic collaterals were opacified. In a female patient with cirrhosis and alcoholic hyaline, the main portal trunk, the inferior mesenteric vein, and the coronary gastric vein were revealed (Fig. 5). In this patient, the contrast medium in the main portal trunk was diluted with the blood flowing from the left portal vein. In a male patient with cirrhosis and alcoholic hyaline, the main portal trunk and the umbilical vein were opacified. Only the intrahepatic portal vein branches were revealed in the other patient with cirrhosis and in 2 patients without cirrhosis. Thus reversal of the portal blood flow or such tendency was shown in cirrhotic patients of Group I.

Clinical course. The female patient of Group I died in hospital from esophageal bleeding. The other patients improved in their clinical and laboratory findings and were discharged.

\section{Discussion}

Though alcoholic hepatitis with alcoholic hyaline had been thought to be rare in Japan (Nakamura et al. 1967), the present study clearly shows that now it is not so rare as previously believed. According to our experience, alcoholic hepatitis with alcoholic hyaline has been found since 1970, and this corresponds to the increase in alcoholic consumption in Japanese.

The daily consumption of alcohol in the present patients was $66 \mathrm{~g}$ or more, and about two-thirds of Group I as well as Group II drank $110 \mathrm{~g}$ or more of alcohol per day. According to Christoffersen et al. (1973), alcoholic intake in cases of alcoholic hepatitis with alcoholic hyaline was more than $50 \mathrm{~g}$ and two-thirds of them were those who consumed more than $150 \mathrm{~g}$ of alcohol per day. Seventyeight per cent of cases of alcoholic hepatitis with and without alcoholic hyaline reported by Lischner et al. (1971) were constant drinkers and consumers in excess of $80 \mathrm{~g}$ of alcohol per day. When the difference of physical constitutions between Japanese and Danish or Americans is considered, the quantity of alcohol consumed by the patients in the present study is not so low as compared with the Danish or American studies.

Most of the patients with alcoholic hepatitis in the present study consumed sake. Whisky or beer was preferred only by a few patients. Therefore, it is clear that sake causes alcoholic hepatitis with alcoholic hyaline like other alcoholic beverages. This is in accord with the concept that alcoholic beverages are harmful in proportion to the quantity of consumed alcohol.

It is interesting that one of the patients with alcoholic hepatitis was a female. Though Japanese women in general do not drink alcoholic beverage so often as European or American women do, a small number of Japanese women, especially 
geisha or similar professional women, consume a large amount of alcohol. Our study shows that some of such women develop alcoholic hepatitis with alcoholic hyaline. It is reported that mortality of alcoholic hepatitis is higher among women than among men (Philips and Davidson 1954). The female patient, geisha, in the present study showed clear evidence of reversal of the portal blood flow and died from esophageal bleeding.

Clinical and laboratory changes did not show significant differences except fever and erythrocyte sedimentation rate between Group I and Group II, though clinical and laboratory changes in general seemed to be more marked in Group I.

Histological study also showed the same tendency. Though liver cirrhosis seemed to be more frequent in patients with alcoholic hyaline than in those without hyaline, the difference was not statistically significant. Statistically significant difference was found only in respect of fatty change. Thus, though hepatic change might be more severe in patients with alcoholic hyaline than in those without hyaline, the difference seems small.

Since both alcoholic hyaline and fatty change are known to disappear after abstinence (Green et al. 1963), the presence of alcoholic hyaline and a higher incidence of fatty change in Group I were at first presumed to be explained by the difference of duration of abstinence prior to biopsy between both groups. However, actual determination denied this presumption. Alcoholic intake was not larger in Group I than in Group II. It cannot be ascribed to the difference of alcoholic beverages, either, because most of the patients of Group I and Group II consumed sake.

As for alcoholic hyaline seen in Group I, it is noted that polymorphonuclear leucocytic infiltration around liver cells containing alcoholic hyaline is not so marked as shown in American and European reports (Harinasuta et al. 1967; Christoffersen 1972; Galambos 1972). Although this may in part be related to rather long duration of abstinence before biopsy in the present study, slightness of leucocytic infiltration in Japanese patients with alcoholic hepatitis was pointed out also by the other investigator (Ito 1976).

The wedged hepatic venous pressure was markedly elevated in alcoholic hepatitis with cirrhosis, and moderately elevated in alcoholic hepatitis without cirrhosis. The elevation in the former is almost the same as that in alcoholic cirrhosis without alcoholic hepatitis estimated in our laboratory. Increase in the wedged hepatic venous pressure in alcoholic hepatitis without cirrhosis was reported also by Reynolds et al. (1969).

The reversal of the portal blood flow or such tendency was shown by wedged hepatic venography in 2 of 5 patients of Group I. According to our experience, such tendency was revealed also in alcoholic cirrhosis without alcoholic hepatitis, but not in non-alcoholic cirrhosis or in patients without cirrhosis (Nakamura et al. 1977). It was found in 5 of 24 cases of alcoholic cirrhosis with or without alcoholic hepatitis, and is thought to show the most severe change in hepatic circulation. It is noteworthy that one of the 2 patients in the present study revealing such 
severe change in hepatic circulation was a female.

\section{References}

1) Christoffersen, P. (1972) Light microscopical feature in liver biopsies with Mallory bodies. Acta path. microbiol. scand., 80, 705-712.

2) Christoffersen, P., Eghöje, K. \& Juhl, E. (1973) Mallory bodies in liver biopsies from chronic alcoholics; A comparative morphological, biochemical, and clinical study of two groups of chronic alcoholics with and without Mallory bodies. Scand.J. Gastroent., $8,341-346$.

3) Galambos, J.T. (1972) Natural history of alcoholic hepatitis; III. Histological changes. Gastroenterology, 63, 1026-1035.

4) Green, J., Mistilis, S. \& Schiff, L. (1963) Acute alcoholic hepatitis. Arch. intern. Med., $112,113-124$.

5) Harinasuta, U., Chomet, B., Ishak, K. \& Zimmerman, H.J. (1967) Steatonecrosis - Mallory body type. Medicine, 46, 141-162.

6) Ito, S. (1976) Origin and clinico-pathological significance of alcoholic hyaline. Saishin Igaku, 31, 2128-2134. (Japanese)

7) Lischner, M.W., Alexander, J.F. \& Galamos, J.T. (197I) Natural history of alcoholic hepatitis; I. The acute disease. Amer. J. dig. Dis., 16, 481-494.

8) Nakamura, S., Takezawa, Y., Sato, T., Aikawa, T. \& Kera, K. (1977) Difference of hepatic circulation between alcoholic and non-alcoholic hepatic eirrhosis. Tohoku $J$. exp. Med., 123, 297-306.

9) Nakamura, T., Nakamura, S., Aikawa, T., Suzuki, O., Onodera, A. \& Karoji, N. (1967) Clinical studies of alcoholic hepatic diseases. Tohoku J. exp. Med., 93, 179-189.

10) Phillips, G.B. \& Davidson, C.S. (1954) Acute hepatic insufficiency of the chronic alcohiolic. Arch. intern. med., 94, 585-603.

11) Reynolds, T.B., Hidemura, R., Michel, H. \& Peters, R. (1969) Portal hypertension without cirrhosis in alcoholic disease. Ann. intern. Med., 70, 497-506.

12) Takada, A. (1976) Alcoholic liver injury in Japan. Saishin Igaku, 31, 2113-2118. (Japanese) 